\title{
Ulrich Heid
}

\section{Korpusbasierte Beschreibung der Variation bei Kollokationen: Deutschland - Österreich - Schweiz - Südtirol}

\begin{abstract}
Kollokationen sind nach unserem Verständnis phraseologische Kombinationen aus zwei Lexemen, die in einer Sprachgemeinschaft konventionalisiert und deren Elemente semantisch ungleichgewichtig sind: Eines wird in derselben Weise verwendet wie in kompositionellen Wortkombinationen (Basis), das andere (Kollokator) erhält seine spezifische Bedeutung nur innerhalb der Kollokation.

Der Artikel diskutiert Möglichkeiten, Kollokationen in Texten zu identifizieren, die für die deutschen Nationalvarietäten aus Deutschland, Österreich, der Schweiz und Südtirol typisch sind. Der Schwerpunkt liegt dabei auf methodischen Fragen: Lassen sich mit einfachen, im Wesentlichen frequenzbasierten Werkzeugen Kollokationsunterschiede zwischen Österreich und Deutschland, der Schweiz und Deutschland, usw. aufweisen? Wie können die Spezifika der Kollokationswahl in den einzelnen Nationalvarietäten anhand von Korpora genauer dokumentiert werden? Sind Varietäten nur in Bezug auf die Kollokatorenwahl spezifisch, oder auch bezüglich morphosyntaktischer Präferenzen (z.B. Artikelgebrauch etc.)?
\end{abstract}

\section{Einleitung}

In diesem Beitrag wird über Methoden und Werkzeuge berichtet, mit denen aus Textkorpora Kollokationskandidaten extrahiert werden können, die man als typisch für die Zeitungssprache von Deutschland (D) bzw. von Österreich (AT), der Schweiz (CH) oder von Südtirol (ST) auffassen kann. Der Schwerpunkt liegt auf Mehrwortausdrücken, die für Österreich, die Schweiz oder Südtirol typisch sind, jedoch in dieser Form im Deutschen von Deutschland selten. Wir ${ }^{1}$ gehen dabei von Zeitungstexten aus, die zum Teil aus einer Vorversion des „Deutschen Referenzkorpus“ des Instituts für Deutsche Sprache stammen. Unsere Materialien sind länderweise geordnet und weisen zwischen ca. 60 Millionen Wörter (für Südtirol) und über 360 Millionen Wörter (für Österreich) auf.

Die hier beschriebenen Arbeiten sind am Institut für maschinelle Sprachverarbeitung der Universität Stuttgart entstanden. Sie sind das Ergebnis der Zusammenarbeit mit Fabienne Cap, Marion Weller und Nadine Siegmund, denen ich für alle Diskussionen und für die praktische Arbeit an den Textdaten herzlich danken möchte. Die Interpretation der Daten (und alle eventuellen Fehlinterpretationen) stammen von mir. 
Als Kollokationen fassen wir phraseologische Kombinationen aus zwei Lexemen auf, die in einer (ggf. regionalen) Sprechergemeinschaft konventionalisiert sind, und die daher in Korpora „auffällig üblich“ sind (Hausmann 1984); ihre Elemente (Hausmann 1979: Basis vs. Kollokator) sind semantisch ungleichgewichtig: Basen haben in der Kollokation dieselbe Bedeutung wie außerhalb, während Kollokatoren eine kollokationstypische Bedeutung haben. Viele Kollokationen haben eine prädikationsartige Struktur (der Kollokator modifiziert die Basis), und fast alle lassen sich einem regelhaften syntaktischen Muster zuordnen; wir untersuchen insbesondere Verb + Objekt-Kollokationen (z.B. CH: Einsitz, nehmen, Entscheid fällen) und Adjektiv + Nomen-Kollokationen (z.B. ST: fixe Stelle, fixer Professor).

Als typisch für eine nationale Varietät fassen wir Wortkombinationen auf, die in Texten einer bestimmten Region proportional häufiger auftreten als in denen einer anderen. Im Sinne von Ammons Polyzentrizitätshypothese (vgl. Ammon 1995 und Ammon/Bickel et al. 2004) nehmen wir stark vereinfachend an, dass die verschiedenen in unserem Korpus enthaltenen Zeitungen aus Deutschland, Österreich, der Schweiz und Südtirol jeweils zusammen so etwas wie eine „nationale Zeitungssprache“ repräsentieren. Bei der Kollokationsextraktion interessieren uns allerdings nicht diejenigen Kollokationskandidaten, deren Basen landestypische „Realien“ bezeichnen $(\mathrm{CH}$ : Kanton, AT: Bundesheer); ebenso nur am Rande diejenigen, die lexikalische Regionalismen enthalten (AT: betrïgerische Krida („Bankrott“), ST: Erbebungen („Ermittlungen“) aufnehmen): Hier ist nur die Kollokatorenwahl interessant (z.B. CH: Zustupf leisten vs. D: Zuschuss geben). Wir zielen stattdessen insbesondere auf „lexikalisch unauffällige“ Kollokationskandidaten: In der Schweiz ist z.B. tiefer Preis prominenter als niedriger Preis.

\section{Fragestellungen - Quellen}

Beim Lesen deutscher Texte aus Österreich, der Schweiz oder Südtirol fallen immer wieder Mehrwortausdrücke auf, die in dieser Form in deutschen Texten aus Deutschland unerwartet sind (vgl. die Beispiele oben in der Einleitung). Wenn es Unterschiede zwischen den einzelnen nationalen Varietäten des Deutschen hinsichtlich der Kollokationsbildung und des Kollokationsgebrauchs gibt, dann sollten diese Unterschiede mit den Mitteln korpuslinguistischer Datenextraktion erhoben und auf diese Weise einer detaillierteren linguistischen Untersuchung zugänglich gemacht werden können.

Eine erste Aufgabenstellung besteht also darin, Kollokationskandidaten aus Textmaterial aus Deutschland, Österreich, der Schweiz und Südtirol zu extrahieren. Für diese Aufgabe stehen uns lediglich Zeitungstexte zur Verfügung. Dabei ist keineswegs ausgemacht, dass der Zeitungstext für die Fragestellung eine besonders geeignete Quelle ist: Viele Meldungen werden von 
internationalen Presseagenturen verbreitet und gegebenenfalls in verschiedenen Ländern ohne Änderungen übernommen; dabei ist es möglich, dass im Sinne der Norm von Deutschland, unauffällige ' Wendungen auch in Österreich, der Schweiz oder Südtirol verbreitet werden. Außerdem haben Journalisten zum Teil ein Interesse daran, eher unmarkierte Texte zu produzieren: Regionalismen, die dem Autor als solche bewusst sind, werden daher unter Umständen eher vermieden. Umgekehrt kann man argumentieren, dass Spezifika, die sich trotzdem in den Zeitungstexten auffinden lassen, unter Umständen vom Autor gar nicht als Regionalismen bemerkt worden sind.

Außerdem ist ein Korpus, welches nur aus Zeitungstexten besteht, natürlich in hohem Maße spezialisiert: Zeitungstext stammt aus einer spezifischen Kommunikationssituation, stellt einen (oder mehrere) spezifische Texttypen und damit auch Sprachverwendungen dar. Vermutlich wäre es sinnvoll, das verwendete Korpus weiter zu untergliedern: Wir berücksichtigen beispielsweise nicht, dass nationale Zeitungen sich vielleicht von regionalen Zeitungen unterscheiden, und dass innerhalb der Schweiz und Österreichs unter Umständen regionale Variation anhand einzelner Regionalzeitungen hätte beobachtet werden können. In dem von uns verwendeten Korpus fehlen die Metadaten, anhand derer eine solche feinkörnigere Analyse angestellt werden könnte.

Ebenso hätte es sich gelohnt, die Zeitungstexte nach Gegenstandsbereichen zu sortieren: Politik, Wirtschaft, Kultur, Sport, Regionalteil. Auch hier wurde keine detaillierte Untergliederung vorgenommen. Eine sorgfältige Studie müsste auch diese Dimension berücksichtigen.

Insgesamt wurden die Zeitungskorpora eher als „Experimentierfeld“ für die entwickelten Werkzeuge benutzt denn als Grundlage für detaillierte Aussagen über die Eigenschaften von Kollokationen des Deutschen von Österreich, der Schweiz oder Südtirol. Auch die Auswertung von Ergebnisdaten ist allenfalls exemplarisch. Geeignete Korpora vorausgesetzt, sollte sich die Methodik aber auf solche Detailuntersuchungen übertragen lassen.

Tabelle 1 stellt die verwendeten Quellen zusammen; die österreichischen und Schweizer Zeitungen aus dem „Deutschen Referenzkorpus“ decken die journalistische Produktion der beiden Länder relativ breit ab, sind aber der Übersichtlichkeit halber nicht einzeln aufgeschlüsselt.

Für Südtirol ist zu bemerken, dass lediglich die Tageszeitung „Dolomiten“ zur Verfügung stand. Wenn wir also Beispiele für „südtiroler Zeitungsprache" geben, so sind diese vielleicht korrekter als Spezifika der Zeitung „Dolomiten“ aufzufassen. ${ }^{2}$

An der Europäischen Akademie Bozen (EURAC) wird im Projekt „Korpus Südtirol“ derzeit ein breiteres und ausgewogeneres Korpus von südtiroler deutschen Texten zusammengestellt und computerlinguistisch erschlossen. Für die „Dolomiten“-Texte danken wir dem Athesia-Verlag, der uns das Material zugänglich gemacht hat, sowie der EURAC, Bozen. 


\begin{tabular}{|l|l|r|}
\hline Land & \multicolumn{1}{|c|}{ Quellen } & Umfang \\
\hline Deutschland & insg. & 258 Mio. \\
& FAZ (1997-1998) & Mio. \\
& Frankfurter Rundschau (1993/1994) & Mio. \\
& Die Zeit (1996-2001) & 51 Mio. \\
& Stuttgarter Zeitung (1993/1994) & 36 Mio. \\
& Handelsblatt (1995/1996) & Mio. \\
\hline Österreich & DeReKo (div. Zeitungen) & 360 Mio. \\
\hline Schweiz & DeReKo (div. Zeitungen) & 183 Mio. \\
\hline Südtirol & Dolomiten (Athesia, EURAC) & 65 Mio. \\
\hline
\end{tabular}

Tab. 1: Datensammlung: verwendete Korpora

Sehr viele Untersuchungen zu Kollokationen konzentrieren sich auf Probleme der Wortkombination: Welche Adjektive oder Verben werden typischerweise mit einem gegebenen Substantiv kombiniert, welche Adverbien mit einem Verb oder Adjektiv usw. Wie unten (Kapitel 3, speziell 3.1.2) gezeigt werden wird, stellen Kollokationen aber insbesondere auch ein interessantes Phänomen im Grenzbereich zwischen Lexik und Grammatik dar, insofern sehr viele Kollokationen spezifische morphosyntaktische Eigenschaften aufweisen, etwa hinsichtlich des Artikelgebrauchs, der Modifizierbarkeit durch Adjektive oder Adverbien, bezüglich Präferenzen für das Auftreten im Aktiv oder im Passiv usw. Wenn man nationale Varietäten untersucht, dann stellt sich auch die Frage, ob sich diese Varietäten primär oder vielleicht ausschließlich auf der lexikalischen Ebene unterscheiden, oder ob es bei gleichem lexikalischem Material auch Unterschiede hinsichtlich solcher morphosyntaktischer Präferenzen gibt. Dieser Frage wird ansatzweise nachgegangen (Abschnitt 4.5); das verfügbare Material reicht allerdings nicht aus, um hier flächendeckende Untersuchungen anzustellen.

Eine weitere Frage betrifft die Methodik des Vergleichs: Stellt sich heraus, dass ein bestimmtes Wortpaar in einer Nationalvarietät typisch und häufig, in einer anderen selten ist, so muss man fragen, durch welches alternative Konstrukt die in der einen Varietät vorhandene Kollokation alternativ in der anderen Varietät ausgedrückt wird. Dies können Kollokationen mit Synonymen sein, wie etwa im Fall von tiefer Preis, welches im Deutschen der Schweiz üblich, im Deutschen von Deutschland eher selten ist.

Es kann aber auch der Fall sein, dass eine Kollokation der einen Varietät durch einen nicht-kollokatorischen Ausdruck der anderen wiedergegeben wird. $\mathrm{Zu}$ fragen ist also, mit welchen Mitteln äquivalente Ausdrücke aus verschiedenen Varietäten miteinander verglichen werden können. Hierfür können bisher lediglich halbautomatische Verfahren auf der Basis einfacher korpuslinguistischer Werkzeuge angeboten werden, aber es ist denk- 
bar, dass Verfahren, die auf die Suche nach Äquivalenten in vergleichbaren Korpora (comparable corpora) angewendet werden, auch hier zum Einsatz kommen können (vgl. Abschnitt 4).

In Abschnitt 3.2 werden einfache Werkzeuge zum Vergleich von Mehrwortdaten in verschiedenen Korpora präsentiert. Diese Werkzeuge sind nicht neu; sie sind in ähnlicher Form für die Extraktion von Fachterminologie aus Fachtexten angewendet worden. Die Frage, die hier diesbezüglich diskutiert werden soll, zielt lediglich darauf, festzustellen, wie weit solche sehr einfachen Werkzeuge für den Varietätenvergleich tragen.

Zusammenfassend lassen sich also die folgenden Fragestellungen festhalten:

- Die lexikalische Selektion in Kollokationen ist präferenziell: Gibt es Präferenzen für die lexikalische Selektion, die als typisch für eine der Nationalvarietäten des Deutschen betrachtet werden können, und wie lassen sich „äquivalente“ Ausdrucksweisen in anderen Nationalvarietäten identifizieren?

- Kollokationen weisen über die lexikalische Selektion hinaus weitere Präferenzen auf, insbesondere im Bereich der morphosyntaktischen Form; untersucht werden soll, ob die Nationalvarietäten auch hier Spezifika aufweisen.

- Aus der Sicht von korpuslinguistischen Datenextraktionswerkzeugen ist zu fragen, inwieweit einfache, im Wesentlichen aus anderen Bereichen der Korpuslinguistik bekannte Extraktionsverfahren auf die Fragestellungen der Variation bei Kollokationen angewendet werden können.

\section{Kollokationen und Kollokationsextraktion aus Texten}

Dieser Abschnitt situiert die vorliegende Studie innerhalb der Kollokationstheorie und stellt dar, mit welchen Verfahren Kollokationskandidaten aus deutschen Texten extrahiert werden.

\subsection{Kollokationsbegriff}

In der Linguistik, Lexikografie und Computerlinguistik ist sehr ausführlich über den Begriff der ,Kollokation“ diskutiert worden. Erst 2004 hat das Institut für Deutsche Sprache seine Jahrestagung und den zugehörigen Sammelband dem Thema der Mehrwortausdrücke gewidmet, mit ausführlichen Diskussionen über Kollokationen (vgl. Steyer (Hg.) 2004).

Für die hier zur Diskussion stehenden Arbeiten ist daher eher eine Positionsbestimmung nötig als eine komplette Aufarbeitung der linguistischen Details (die den vorliegenden Rahmen sprengen würde, vgl. dazu neu z.B. Forkl 2010 und Tutin 2010). 


\subsubsection{Die Arbeitsdefinition von Bartsch}

Ausgangspunkt für die hier diskutierten Arbeiten ist eine computerlinguistische Interpretation des von Hausmann so genannten lexikografischdidaktischen Kollokationsbegriffs (vgl. Hausmann 2004); dieser ist in einer der Computerlinguistik nahen Form von Bartsch (2004, S. 76) zusammengefasst worden: „Collocations are lexically and/or pragmatically constrained recurrent cooccurrences of at least two lexical items which are in a direct syntactic relation with each other."

Diese „Arbeitsdefinition“ (Bartsch) enthält eine Reihe von definitionsrelevanten Aspekten, die in den folgenden Abschnitten kurz aufgelistet und interpretiert werden.

Wir bedienen uns dabei der Hausmann'schen Terminologie, die Basis und Kollokator unterscheidet (Hausmann 1979, 2004): In einer Kombination wie (einen) Hund ausführen, (eine) Frage stellen oder scharfe Kritik sind Hund, Frage und Kritik als (im Sinne von Hausmann autosemantische) Basen zu verstehen: Ihre Bedeutung ist innerhalb und außerhalb der Kollokation dieselbe. Die Verben ausfübren und stellen, bzw. das Adjektiv scharf sind Kollokatoren: Sie erhalten ihre Bedeutung erst innerhalb der Kollokation: stellen in Frage stellen dient dazu, eine allgemeine „Realisierung“ auszudrücken (Mel'čuk würde hier die Lexikalische Funktion, oper(ari)'benutzen, vgl. Mel'čuk et al. 1984); scharf in scharfe Kritik dient als Ausdruck eines hohen Grads an Kritik, ähnlich wie heftig oder harsch in heftige Kritik, harsche Kritik. Die Bedeutungen von stellen oder scharf sind also kollokationsspezifisch und nicht notwendig aus den Bedeutungen dieser Lexeme außerhalb der Kollokationen ableitbar; bei Hund ausführen liegt der Fall ähnlich, auch wenn ausführen spezifischer ist als die eher allgemeinen Bedeutungen von scharf oder stellen.

\subsubsection{Morphosyntaktische Idiomatisierung in Kollokationen}

Wenn Bartsch sagt, dass Kollokationen „lexically and/or pragmatically constrained" sind, dann verweist sie damit auf die u.a. auch von Hausmann (2004) hervorgehobene lexikalische Auswahl: Zur sprachlichen Realisierung des Konzepts des Spazierenführens eines Hunds gehört das Verb ausfübren, nicht z.B. (?) Hund führen, (?) Hund spazierenführen.

Diese lexikalische Auswahl interpretieren wir als ein Stück Idiomatisierung. Hierzu gehört auch die Festlegung morphosyntaktischer Präferenzen: Bei Frage + stellen kann Frage in beliebigen Nominalphrasen vorkommen (eine wichtige Frage stellen; die Fragen stellen, die keiner hören will; Fragen stellen, auf die es keine Antwort gibt; usw.); bei der Kollokation in + Kraft + treten ist dagegen die Morphosyntax der Nominal- bzw. Präpositionalphrase sehr eingeschränkt: Man hat die Kollokation nur im Singular und ohne Artikel, also in 
Kraft treten, aber nicht (?) in die Kräfte treten usw. (Wo wir bewusst die Determination in einer Kollokation unberücksichtigt lassen wollen, schreiben wir „+“ zwischen Basis, Kollokator und ggf. Präposition.)

Die morphosyntaktische Idiomatisierung (oder: formale Fixiertheit) ist für die Interpretation von Kollokationen konstitutiv, ähnlich wie für idiomatische Wendungen. Dementsprechend gibt es auch Fälle, wo zwei Lesarten sich durch die Morphosyntax, nicht aber durch die am Mehrwortausdruck beteiligten Lexeme unterscheiden (in Gang kommen vs. in die Gänge kommen), oder wo der Unterschied zwischen wörtlicher und idiomatischer Interpretation mit den morphosyntaktischen Eigenschaften einhergeht (in die Hand nebmen (,übernehmen, lenken') vs. (etw.) in die Hand/ in die Hände nehmen (,anfassen')). Die morphosyntaktische Fixiertheit (bzw. die ggf. eingeschränkte morphosyntaktische Variabilität) von Kollokationen ist ein Phänomen an der Schnittstelle von Lexikon und Syntax; das (ggf. teilweise) Fehlen der üblichen syntaktischen Veränderungsmöglichkeiten von Phrasen ist Teil der Idiomatisierung. Im Hinblick auf die Analyse der Unterschiede zwischen den Varietäten des Deutschen ist zu fragen, wie stabil diese morphosyntaktischen Spezifika quer durch die untersuchten Varietäten sind.

Nicht alle Kollokationen weisen eine starke morphosyntaktische Fixiertheit auf. Die Kollokation Frage + stellen kann gerade in allen Formen verwendet werden, die für beliebige Nominalphrasen möglich sind; hier ist nur die Selektion von stellen (neben z.B. aufwerfen) typisch und idiomatisch. Die Kollokatorenwahl ist aber wiederum aus semantischen Prinzipien nicht vorhersagbar (und sprachspezifisch, vgl. ask a question, poser une question usw.), d.h. „lexically determined“ im Sinne von Bartsch (2004).

\subsubsection{Rekurrenz von Kollokationen}

Bartsch spricht davon, dass Kollokationen „recurrent" seien; die auch schon von Hausmann (1984) beobachtete ,auffällige Üblichkeit' der Kollokationen hat in der Computerlinguistik dazu geführt, dass statistische Assoziationsmaße dazu eingesetzt wurden, Kollokationskandidaten aus Texten zu extrahieren. Wenn zwei Wörter proportional häufiger miteinander vorkommen als statistisch erwartbar, dann werden sie von den Assoziationsmaßen identifiziert und extrahiert. Evert (2005) hat die Statistik dieser Maße untersucht und analysiert, inwieweit sie mit der linguistisch-lexikografischen Intuition über Kollokationen korrelieren. Als erster Anhaltspunkt sind einige der Maße sehr nützlich; ihr relativ enger Zusammenhang mit der Wortfrequenz kann allerdings mitunter zu Problemen führen. Wir benutzen auch für die vorliegende Studie Assoziationsmaße, insbesondere die Werte des „Log Likelihood Ratio Tests“ (Dunning 1993). 
Der Test vergleicht die beobachtete Häufigkeit der Kollokationen mit der statistisch erwartbaren Häufigkeit, unter Einbezug von Daten zu anderen Kombinationen der beiden Kollokationsbausteine. Es handelt sich um einen statistischen Hypothesentest, der ermittelt, wieviel Evidenz gegen die Annahme vorliegt, der jeweilige Kollokationskandidat sei eine Zufallskombination.

\subsubsection{Binarität und syntaktische Relation in Kollokationen}

Bartsch sagt, dass Kollokationen aus mindestens zwei Elementen bestehen; diese Annahme ist konsistent mit der Unterscheidung in Basis und Kollokator, wie sie Hausmann trifft; viel diskutiert wurde in den letzten Jahren über die Frage, welche Länge Kollokationen annehmen können. Dabei wurden Mehrwortausdrücke als Kollokationen diskutiert, die mehr als zwei Lexeme enthalten. Deutlich ist auf jeden Fall, dass Mehrwortausdrücke selbst Teil von Kollokationen sein sowie mehrere Kollokationen kombiniert auftreten können (z.B. scharf + Kritik, kombiniert mit Kritik + üben in scharfe Kritik üben, vgl. Heid 1994). Für die Untersuchungen im vorliegenden Rahmen zielen wir primär auf Kollokationskandidaten aus zwei Lexemen.

Kollokationen unterliegen den Regeln der Syntax; Bartsch folgt hier dem von Evert (2005) eingeführten Begriff der ,relationalen Kookkurrenz': Zwischen den Elementen der Kollokation gibt es eine grammatische Relation; diese kann parallel zu einer Modifikationsrelation im Sinne der Phrasenstrukturgrammatik liegen (z.B. bei Adjektiv + Nomen-Kollokationen, scharfe Kritik, oder bei Adverb + Adjektiv-Kollokationen, tief rot), sie kann aber auch über Phrasengrenzen hinweggehen (z.B. bei Subjekt + Verb-Kollokationen, die Frage stellt sich, Angst kommt auf, usw.).

Die Anzahl der grammatischen Relationen, die kollokationsträchtig sind, ist relativ begrenzt; die Relationen werden in unserer Extraktionsarbeit als Modell für die Suche nach Lexempaaren genommen; wir suchen z.B. nach Paaren aus Verb und Objekt, aus Verb und Präpositionalobjekt, aus Adjektiv und Nomen usw. Anders als in der Idiomatik, wo zum Teil idiosynkratische grammatische Strukturen auftreten (welche Art Phrase ist z.B. EN by and large?), halten wir die Festlegung auf ein vorab definiertes Inventar von grammatischen Typen bei der Suche nach Kollokationen nicht für problematisch; ausgehend von den einfachen Paar-Modellen kann in einem weiteren Schritt nach Kombinationen von Kollokationen gesucht werden (vgl. Heid/Weller 2010).

\subsubsection{Aktuelle Forschungen zum Kollokationsbegriff}

Die Arbeit von Bartsch (2004) wurde hier zusammen mit den Publikationen von Hausmann (insbesondere Hausmann 2004) als Grundlage für die Einordnung unseres Kollokationsbegriffs verwendet. Bei Bartsch und Haus- 
mann finden sich die folgenden definitionsrelevanten Elemente: (i) phraseologischer Charakter (,lexically and/or pragmatically determined“), (ii) Konventionalisiertheit (,recurrent"), (iii) Binarität (,at least two elements"), (iv) Asymmetrie und Semi-Kompositionalität (Hausmann: Basis vs. Kollokator) und (v) syntaktische Relation.

Neueste Arbeiten von Tutin (2010) und von Forkl (2010) gehen in eine ähnliche Richtung; statt von Semi-Kompositionalität spricht Forkl davon, dass Kollokationen unidirektional kompositionell sind: Man versteht sie, kann sie aber ohne Vorwissen nicht korrekt bilden. Diese Eigenschaft unterscheidet Kollokationen von idiomatischen Wendungen, die sowohl in der Sprachproduktion, als auch in der Rezeption ohne Vorwissen nicht verarbeitbar sind. Tutin und Forkl ergänzen das obige Inventar von Definitionsbausteinen um die Tatsache, dass in Kollokationen eine prädikationsartige Relation existiert: Der Kollokator fungiert in der Regel als Prädikat, die Basis als Argument. Tutin spricht von „structure prédicat/argument“, Forkl sagt, dass Kollokationen relationsprädikativ sind.

Dieser kurze Überblick zeigt, dass mehrere Faktoren bei der Definition von Kollokationen eine Rolle spielen; diese Faktoren sind genauso für die Datenextraktion aus Textkorpora relevant. Wie sie in Suchverfahren umgesetzt werden, wird im folgenden Abschnitt dargestellt.

\subsection{Kollokationsextraktion}

\subsubsection{Ansätze}

Hier kann aus Platzgründen kein vollständiger Überblick über Verfahren der Kollokationsextraktion gegeben werden (vgl. dazu z.B. Heid 2009; Evert 2005 usw.). In starker Vergröberung kann man zwischen symbolischen und statistischen Verfahren unterscheiden.

Die statistischen Verfahren nehmen insbesondere das Kriterium der Rekurrenz (der ,auffälligen Üblichkeit') der Kollokationen als Suchkriterium; extrahiert werden Wortpaare, die häufiger als statistisch erwartet miteinander auftreten, sei es adjazent oder in einem bestimmten ,Textfenster', z.B. im Abstand von drei Wörtern. Solche ,positionelle' Kookkurrenz (Evert 2005) ist z.B. im Werkzeug „Wordsmyth“ in reiner Form implementiert.

Symbolische Verfahren setzen auf dem Aspekt der relationalen Kookkurrenz auf: Gesucht werden z.B. alle Paare aus Adjektiven und (Bezugs-) Nomina, oder aus Verben und ihren Objektsnomina. Extrahiert man in der Tat alle derartigen Paare, dann erhält man natürlich auch sehr viele, die nicht rekurrent und auch nicht idiomatisch sind.

Folglich wurde vielfach mit Kombinationen beider Verfahrenstypen gearbeitet. Smadja (1993) hat als erster statistische und symbolische Ansätze kombiniert: erst eine statistische Suche nach allen signifikanten WortpaarAssoziationen, dann ein Filter nach Wortartkombinationen. Für das Deut- 
sche wurde der umgekehrte Weg vorgeschlagen: erst Extraktion aller Paare einer bestimmten grammatischen Relation (z.B. Verben und ihre Objektsnomina), dann eine Sortierung dieser Kandidaten nach der Stärke der Assoziation (Heid 1998; Krenn 2000; Evert 2005). Dieses letztere Verfahren ist z.B. auch in der „Sketch Engine“ von Kilgarriff et al. (2004) für das Englische implementiert. „Sketch Engine“ ist ein kommerzielles Werkzeug zur Analyse von Korpora, das die Funktionen einer Konkordanz mit denen der Suche nach Wortpaaren kombiniert. Der Benutzer kann zwischen beidem hin- und herspringen, z.B. um sich Konkordanzzeilen zu einer Wortkombination anzeigen zu lassen (vgl. http://sketchengine.co.uk/ (Stand: 13.12.2010) zu Details und eine Möglichkeit zum Ausprobieren des Werkzeugs).

Neuere Verfahren kombinieren die letztgenannte Strategie mit einer Suche nach denjenigen Paaren, die morphosyntaktische Fixiertheit zeigen (Fazly/Stevenson 2006); damit werden idiomatische Wendungen extrahiert, sowie derjenige Teil der Kollokationen, der starke morphosyntaktische Präferenzen aufweist; weniger geeignet ist dieses (im Kern eher auf Idiomatik ausgerichtete) Verfahren für Kollokationen vom Typ Frage + stellen, die keine morphosyntaktischen Präferenzen aufweisen.

\subsubsection{Spezifische Probleme der Extraktion deutscher Kollokationen}

Für das Englische erzielt die „Sketch Engine“ (Kilgarriff et al. 2004) Resultate, die mit der lexikografischen Intuition über Kollokationen (,welche der gefundenen Kombinationen sind potenziell wörterbuchrelevant?', vgl. Kilgarriff et al. 2010) relativ gut übereinstimmen. Die Extraktion in „Sketch Engine" erfolgt mit einer relativ einfachen flachen Grammatik, die mit Wortart- und Lemmaangaben annotierte Texte als Eingabe nimmt. Diese flache Analyse genügt für das Englische, weil eine statistisch relevante Menge von Belegen in der für das Englische normalen konfigurationalen Abfolge von Subjekt, Verb und Objekt vorkommt. Sucht das System also Nomina links vom flektierten Verb, so findet es typische Subjekt + VerbKollokationen, sucht es vom flektierten Verb ausgehend nach rechts, findet es Verb + Objekt-Kollokationen.

Weitere, relativ erfolgreiche Experimente mit „Sketch Engine“ wurden für das Slowenische und das Japanische durchgeführt (vgl. Kilgarriff et al. 2010); beide Sprachen haben relativ explizite und wenig ambige Markierungen der grammatischen Relationen (Subjekt, Objekt, usw.), entweder durch morphologische Kasusendungen (im Slowenischen) oder durch Partikeln (im Japanischen).

Die Extraktion aus deutschen Texten macht mehr Schwierigkeiten. Eine flache Analyse nach dem Muster der „Sketch Engine“ muss mindestens die drei Modelle der Konstituentenstellung des Deutschen berücksichtigen (Verb-erst, Verb-zweit, Verb-letzt): Es müssen also mehr Suchmuster 
bereitgestellt werden, als für das Englische. Die bloße Permutation der Suchmuster genügt aber nicht für eine qualitativ hochwertige Extraktion: Aufgrund des weit verbreiteten Kasus-Synkretismus lässt sich nicht ohne Weiteres feststellen, welche Nominalphrasen Subjekte und welche Objekte sind; selbst Dativobjekte sind bei artikellosen Nominalphrasen (z.B. das genügt Anforderungen von Arbeitgebern wie Arbeitnehmern) oft nicht kasusmarkiert.

Die Kombination von Kasussynkretismus, alternativen Wortstellungsmodellen und relativ freier Wortstellung im deutschen Mittelfeld führt dazu, dass ein flacher Ansatz auf der Basis von Korpusdaten mit Wortart- und Lemmaannotation allein für die Extraktion deutscher Kollokationen eher wenig überzeugende Resultate liefert (vgl. Ivanova et al. 2008). Evert (2004) hat anhand der Negra-Baumbank ermittelt, dass nur knapp über 20\% der Nominalphrasen eines Zeitungstexts kasus-eindeutig sind.

\subsubsection{Kollokationsextraktion auf der Basis tiefer Analyse}

Die oben dargestellte Problemlage suggeriert die Suche nach Alternativen zur flachen Kollokationsextraktion im Sinne von „Sketch Engine“. Wir benutzen daher Korpora, die mit einer tiefen syntaktischen Analyse annotiert sind, die von einem dependenzgrammatischen Analysewerkzeug geliefert wird. Wir verwenden Schiehlens (2003) Parser FSPar („Finite State Parser"), ein Werkzeug, das grammatische Funktionen, aber auch morphosyntaktische Merkmale ausgibt. Wenn ein Satz Wörter oder Wortfolgen enthält, deren grammatische Funktion nicht eindeutig identifiziert werden kann (Etiketten-Ambiguität, z.B. Subjekt vs. direktes Objekt), so wird die Mehrdeutigkeit markiert; ebenso bei Zuordnungsambiguitäten (z.B. PP-Attachment). Die Ausgabe des Parsers ist also (durch Angabe von Alternativen) explizit unterspezifiziert.

Für die Kollokationsextraktion hat die unterspezifizierte Ausgabe den Vorteil, dass z.B. Sätze mit Etikettenambiguitäten (Subjekt vs. Objekt) gezielt aus der Menge der Rohdaten für die Extraktion ausgeschlossen werden können; im Fall von Attachment-Ambiguitäten gehen wir davon aus, dass die Datenmenge eventuell vorhandene Fehlzuordnungen ausgleicht: Sie werden bei der Extraktion als unkritisch angesehen und ,überlesen'.

Tabelle 2 und die zugehörige Abbildung stellen die Analyse eines einfachen Satzes dar (die zweite Studie lieferte ähnliche Ergebnisse), und zwar einmal als Dependenzstrukturgraf sowie zweitens als Tabelle; die Tabellenform kann mit einfachen Mitteln automatisch durchsucht werden, und zwar nach grammatischen Relationen (Adjektiv + Nomen: ähnliche Ergebnisse, Verb + Objekt: Ergebnis + liefern, usw.), aber auch nach den morphosyntaktischen Merkmalen der einzelnen Kollokationsbausteine; in der fünften Spalte der Tabelle sind die vom Analysewerkzeug gefundenen morphosyntaktischen Eigenschaften explizit angegeben: Sie brauchen nur von dort extrahiert zu 
werden. Spalte 1 der Tabelle enthält die fortlaufende Position der Wörter im Satz (beginnend mit der Nummerierung , $0^{\circ}$ ), Spalte 2 die Wortformen, Spalte 3 die Wortart-Etiketten des Stuttgart-Tübingen-Tagsets (dabei sind ADJA: Attributives Adjektiv, ART: Artikel, NN: Nomen, VVFIN: finites Vollverb). In Spalte 4 sind Lemmaangaben zu finden (dabei wird ,d- ' für alle Formen des bestimmten Artikels gewählt), in Spalte 5 morphosyntaktische Merkmale (hier in Auswahl dargestellt). Die grammatischen Relationen zwischen den Wörtern sind in der letzten Spalte notiert: Beim Nomen Ergebnisse (Zeile 5, d.h. das Wort, das an sechster Stelle im Satz steht) ist z.B. die Funktion des Objekts (,NP:akk') vermerkt; in der zweitletzten Spalte findet sich das Bezugswort für die grammatische Relation, im Fall von Ergebnisse das Wort mit der Positionsnummer 3 (d.h. das Verb liefern); die Positionsnummer , $-1^{\text {‘ }}$ wird für den Wurzelknoten des Dependenzbaums, für Satzendezeichen und für Teilstrukturen vergeben, die das Analysewerkzeug nicht in der Dependenzstruktur verorten kann.

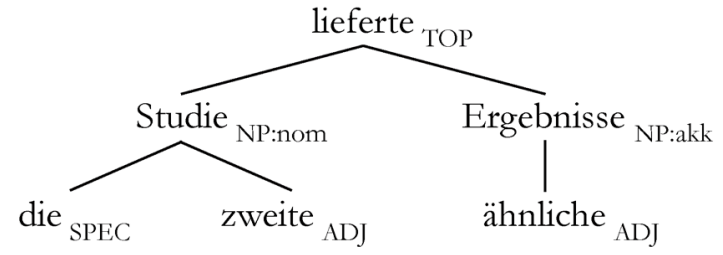

\begin{tabular}{|c|l|l|l|l|c|l|}
\hline Position & Wortform & POS & Lemma & Morphol. & Bezugswort & $\begin{array}{c}\text { gramm. } \\
\text { Funktion }\end{array}$ \\
\hline 0 & Die & ART & d- & & 2 & SPEC \\
\hline 1 & zweite & ADJA & zweite. & & 2 & ADJ \\
\hline 2 & Studie & NN & Studie & Nom:F:Sg & 3 & NP:nom \\
\hline 3 & lieferte & VVFIN & liefern & 3:Sg: Past:Ind & -1 & TOP \\
\hline 4 & ähnliche & ADJA & ähnlich & & 5 & ADJ \\
\hline 5 & Ergebnisse & NN & Ergebnis & Akk:N:Pl & 3 & NP:akk \\
\hline 6 & \$. & \$. &. & & -1 & TOP \\
\hline
\end{tabular}

Tab. 2: Dependenzbaum für die zweite Studie lieferte ähnliche Ergebnisse und tabellarische Darstellung der Dependenzstruktur

Zusammenfassend lassen sich folgende Merkmale (und gleichzeitig Vorteile) der dependenzbasierten Datenextraktion festhalten: Der Dependenzparser verwendet wesentlich mehr grammatisches Wissen als der „Sketch Engine“-Ansatz; er liefert daher auch detailliertere Ergebnisse; diese enthalten u.a. eine vollständige morphosyntaktische Beschreibung. Die Ergebnisrepräsentation ist unterspezifiziert, so dass Kasusambiguitäten als potenzielle Fehlerquelle bei der Kollokationsextraktion identifiziert und ausgefiltert 
werden können; das erhöht die Präzision der Extraktionsergebnisse. Da alle deutschen Wortstellungsmuster abgedeckt sind, erhöht sich auch der Recall (die Ausbeute) gegenüber einer flachen, pattern-basierten Grammatik (vgl. Heid et al. 2008).

\subsubsection{Linguistische Klassifikation der extrahierten Kollokationskandidaten}

Die oben diskutierten Extraktionsverfahren nutzen den Definitionsaspekt der relationalen Kookkurrenz, indem sie z.B. Verb + Objekt-Paare identifizieren. Außerdem wird der Aspekt der Rekurrenz überprüft, insofern die extrahierten Kandidatenmengen nach dem Log-Likelihood-Wert (absteigend) sortiert werden.

Darüber hinaus nutzen wir auch den Aspekt der morphosyntaktischen Fixiertheit: Die morphosyntaktischen Parameter jedes zur Extraktion benutzten Satzes werden einzeln notiert. Anders als Fazly/Stevenson (2006) verwenden wir aber die morphosyntaktischen Präferenzen der extrahierten Daten nicht als Entscheidungskriterium über idiomatische vs. nicht-idiomatische Kookkurrenzen, sondern wir bewahren die Daten auf, um sie nachfolgend statistisch auszuwerten: Wie stabil sind einzelne Präferenzen? Wieviel Flexibilität findet sich in den analysierten Texten?

\begin{tabular}{|l|l|}
\hline v_lemma & vor\#nehmen \\
reflexiv & - \\
negation & - \\
subjekt & Pharmakologe,Arzt \\
subjekt_num & Pl,Pl \\
akk_objekt_n_lemma & Bewertung \\
akk_objekt_determiner & indef \\
akk_objekt_adjektiv & kritisch \\
akk_objekt_num & Sg \\
prep & in \\
prep_nn & in:Bericht \\
fusion & - \\
satzbeleg & In dem seit 1984 jährlich erscheinenden \\
& Bericht nehmen Pharmakologen und \\
& Ärzte eine kritische Bewertung der Arz- \\
korpus & neimittel vor. \\
\hline
\end{tabular}

Tab. 3: Auszug aus der Kollokationsdatenbank: ein Satz und die daraus extrahierten Merkmale

Tabelle 3 stellt einen Auszug aus der Datenbank dar, in der die einzelnen Belegsätze gesammelt und mit den automatisch annotierten morphosyntaktischen Kriterien zusammen abgelegt werden. In der Datenbank können 
auch Adjektive, Adverbien und andere Kontextpartner der Kollokationen aufgesammelt und ausgewertet werden; eine mögliche Beispielanfrage könnte lauten: Bezogen auf alle Okkurrenzen von Bewertung + $(A D J)$ ? + vornehmen, wie häufig ist das Adjektiv kritisch, d.h die Kollokationskombination eine kritische Bewertung (von ...) vornebmen?

Die Auswertung der Datenbankeinträge erfolgt in der Regel nach Basis + Kollokator-Paaren; so kann z.B. festgestellt werden, dass Bewertung + vornehmen primär im Singular, häufiger mit indefinitem als mit definitem Artikel und oft mit Adjektiven wie endgültig, abschließend, kritisch vorkommt.

Für die Untersuchung der Kollokationen aus Texten aus Deutschland, Österreich, der Schweiz und Südtirol werden die Korpora einzeln nach den oben genannten Verfahren analysiert; für jedes Korpus werden die Daten in der Datenbank abgelegt; im nächsten Schritt müssen sie untereinander, nach den vier Sprachräumen, verglichen werden.

\section{Methoden und Probleme des Vergleichs von Varietäten bezüglich Kollokationen}

Wie oben angedeutet, sollen einfache Werkzeuge für den Vergleich von Kollokationsunterschieden zwischen den deutschen Varietäten benutzt werden. Die Frage, die hier ansatzweise mitbeantwortet werden soll, ist, wie weitgehend sich mit solchen einfachen Werkzeugen ein solcher Vergleich überhaupt realisieren lässt.

\subsection{Ein einfaches frequenzbasiertes Verfahren für den Varietätenvergleich}

Ein besonders einfaches Hilfsmittel für den lexikalischen Vergleich von Texten ist der Vergleich der (relativen) Frequenz von Wörtern in den verschiedenen Texten. Ein solcher Vergleich erlaubt es, Wörter (oder, in unserer Erweiterung, Wortkombinationen) zu identifizieren, die in einem gegebenen Text häufig, in einem anderen seltener sind. Ahmad et al. (1992) haben solche Berechnungen dazu eingesetzt, Fachtermini aus Fachtexten zu extrahieren.

Die zu Grunde liegende Idee ist sehr einfach: Wörter, die in einem Fachtext wichtig sind, kommen dort öfter vor als in einem Text oder Textkorpus, das keine fachliche Bindung aufweist. Man zählt also die Okkurrenzen jedes Wortes aus dem Fachtext und bestimmt außerdem die Häufigkeit aller Fachtext-Wörter im Vergleichstext. Da beide Texte unterschiedlich umfangreich sein können, dividiert man jeweils durch die Gesamtzahl der Wörter des betreffenden Texts (ähnlich wie beim Berechnen von parts-permillion). Dies ergibt zwei Zahlen, die zueinander in Relation gesetzt wer- 
den (dividiert: Fachtext-Frequenz/Gemeinsprachtext-Frequenz). Es ergibt sich ein Quotient, der angibt, wievielmal häufiger das betreffende Wort im Fachtext vorkommt als im Gemeinsprachtext.

Ahmad et al. (ebd.) nennen den Quotienten die „weirdness ratio“, weil er angibt, wie stark die Verteilung einzelner Wörter in einem Fachtext gegenüber der Frequenz in einem nicht-fachlichen Text „verschoben“ (weird) ist. Kommt das untersuchte Fachwort im Gemeinsprachtext überhaupt nicht vor, so entsteht ein unendlich großer Quotient (inf, man kann nicht durch Null dividieren), d.h. man bekommt für jedes Textpaar als Ergebnis des Verfahrens zwei Listen: diejenigen der Wörter, die nur Teil des Fachtexts sind, und zweitens eine Liste derjenigen, die in beiden Texten auftreten, aber im Fachtext häufiger sind. Die Elemente beider Listen sind, mindestens im oberen Frequenz- bzw. Quotientenbereich, relativ gute Fachwort-Kandidaten. Die Tabellen 4 und 5 zeigen die Ergebnisse der Analyse eines Korpus, das die Europäische Pharmabehörde EMEA als Sammlung von Medikamentenprüfberichten bereitgestellt hat. In unseren Experimenten wurde dieser Text mit einem Ausschnitt aus der Frankfurter Rundschau verglichen, den wir vereinfachend als nicht fachspezifisch betrachten.

In Tabelle 4 sind die zehn häufigsten Wörter vermerkt, die nur in den EMEA-Texten vorkommen. Tabelle 5 enthält zehn Wörter, die zwar auch im Vergleichstext (hier: die Jahrgänge 1993/94 der „Frankfurter Rundschau“, 40 Millionen Wörter) vorkommen, aber im EMEA-Korpus (10 Millionen Wörter) proportional viel häufiger sind (auch hier: top-10 nach Quotient).

Das dargestellte Suchverfahren kann (mit gewissen Einschränkungen) auf Texte beliebiger Größe angewendet werden; die Texte sollten nicht von allzu unterschiedlicher Größe sein. Außerdem lässt es sich statt auf einzelne Wortformen auf Lemmazahlen (aufsummiert aus den einzelnen Okkurrenzen der Wortformen) und auf Wort- bzw. Lemma-Paare anwenden (auch hier ist aus Sicht der Lexikonstatistik zum Teil allerdings Vorsicht geboten).

$\mathrm{Da}$ es sich um ein rein rechnerisches Verfahren handelt, ist es auf beliebige Fragestellungen anwendbar: Wir haben daher die Ahmad'schen ,weirdness"-Zahlen für Schweizer bzw. für österreichische Texte ermittelt, jeweils mit den Zeitungstexten aus Deutschland als Vergleichstext. Das Verfahren wenden wir sowohl für Einzelwörter, als auch für Kollokationen an.

Für Adjektiv + Nomen-Kollokationen ergeben sich so z.B. die Kandidaten heurig + Jahr (heuriges Jahr, im heurigen Jahr, ...) als hochfrequent in den österreichischen Zeitungen, aber nicht vertreten im Material aus Deutschland. In ähnlicher Weise finden sich regierender Meister oder regierender Weltmeister in unseren österreichischen Texten 207-mal bzw. 193-mal häufiger als in unseren Daten aus Deutschland, wo die Wendung extrem selten ist. Die in Deutschland geläufige Kollokation amtierender (Welt-)Meister ist in den österreichischen Texten nur unerheblich häufiger (1,7 bzw. 2,9-mal) als in den deutschen Texten aus der Bundesrepublik. Diese kleinen Unterschiede 
gehen darauf zurück, dass die Proportionen von Sport-Texten gegenüber Texten über Wirtschaft, Kultur, Politik, etc. für die nationalen Teilkorpora nicht exakt gleich (und auch nicht im Detail bekannt) sind. Aus methodischer Sicht würde eine detailliertere Studie voraussetzen, dass auch die Verteilung von Kollokationen über die Domänen (d.h. thematischen Teile) der Zeitungen mit erhoben und in den Berechnungen berücksichtigt wird.

\begin{tabular}{|l|c|}
\hline \multicolumn{1}{|c|}{ Termkandidat } & Frequenz \\
\hline Durchstechflasche & 5638 \\
Injektionsstelle & 3489 \\
Pharmakokinetik & 3426 \\
Hämoglobinwert & 3395 \\
Fertigspritze & 3271 \\
Ribavirin & 3234 \\
Gebrauchsinformation & 2801 \\
Dosisanpassung & 2580 \\
Epoetin & 2302 \\
Hydrochlorothiazid & 2128 \\
\hline
\end{tabular}

Tab. 4: Fachausdrücke, die nur im EMEA-Korpus gefunden wurden (nach Frequenz, absteigend, top-10)

\begin{tabular}{|l|c|c|}
\hline Termkandidat & Quotient & Frequenz \\
\hline Filmtablette & 25522 & 6389 \\
Injektionslösung & 19854 & 4970 \\
Packungsbeilage & 14710 & 7365 \\
Niereninsuffizienz & 14233 & 3563 \\
Verkehrstüchtigkeit & 13558 & 3394 \\
Leberfunktion & 8385 & 2099 \\
Hypoglykämie & 8353 & 2091 \\
Toxizität & 7957 & 1992 \\
Einnehmen & 7035 & 7045 \\
Hypotonie & 6823 & 1708 \\
\hline
\end{tabular}

Tab. 5: Fachausdrücke aus dem EMEA-Korpus, die auch im gemeinsprachlichen Vergleichskorpus (Frankfurter Rundschau, 1993/1994) vorkommen (nach der „weirdness-ratio“, absteigend, top-10)

Eine Prüfung unserer Texte aus der Schweiz und aus Südtirol ergibt übrigens, dass amtierender (Welt-)Meister in beiden Gebieten üblich und häufig ist; allerdings findet sich regierender (Welt-)Meister (erwarteterweise) auch in Südtirol (allerdings viel weniger prominent als in Österreich), in den Schweizer Texten dagegen überhaupt nicht. Also handelt es sich wohl um einen Austriazismus. 
Das oben beschriebene Verfahren erlaubt es also, Frequenzhelvetismen, -Austriazismen usw. zu identifizieren, und zwar für Einzelwörter und für Kollokationen. Wir berechnen für letztere die Häufigkeiten der lemmatisierten Paare.

\subsection{Klassifizierung von varietätenspezifischen Kollokationen}

Das oben diskutierte Verfahren wirft alle Einzelwörter und Wortpaare auf, deren Frequenz in einem der verglichenen Texte deutlich höher ist als im anderen. Will man diese Daten aus varietätenlinguistischer Sicht interpretieren, so muss eine Klassifizierung in verschiedene Subtypen vorgenommen werden.

Ein Grund für eine Klassifizierung liegt darin, dass bestimmte Lexeme, die in den Kollokationen vorkommen, auch alleine, ohne die jeweilige Kollokation, für einen bestimmten geographischen Raum spezifisch sind. Beispiele sind „regionale Realien“, wie z.B. CH Franken, AT Bundesheer, ST Landesrat. Dass diese Wörter in Schweizer, österreichischen oder südtiroler Texten eine proportional erheblich höhere Häufigkeit haben als in Texten aus Deutschland, ist trivialerweise durch die Regionen gegeben, über die die jeweiligen Zeitungen schreiben. Analoges gilt für regionale idiomatische Ausdrücke (z.B. CH ein Nein in die Urne legen), oder für regionale MehrwortTermini (z.B. des Rechts oder der Verwaltung, vgl. ST landwirtschaftliches Grün, $\mathrm{CH}$ blaue Zone). Solche Kombinationen und auch Kollokationen mit Wörtern, die Realien bezeichnen, sind nicht Ziel der Extraktion.

Eine zweite Kategorie von Kombinationen ist ebenfalls nur bedingt interessant, auch wenn es sich dabei um Kollokationen im Sinne der obigen Definition handelt. Beispiele sind AT betrügerische Krida, CH bängiges Verfahren. In diesen Fällen ist die Basis (z.B. Krida) oder der Kollokator (z.B. bängig) ein spezifisches Lexem der jeweiligen Nationalvarietät (Krida für Insolvenæz, hängig für anhängig).

Dass wiederum solche Fälle vom statistischen Verfahren als massiv vom Gebrauch in Deutschland abweichend identifiziert werden, liegt auf der Hand: Krida und bängig kommen in den Texten aus Deutschland kaum vor. Aus Kollokationssicht sind diese Fälle aber nur bedingt interessant; in betrügerische Krida wird der Kollokator betrügerisch (der keine Varietätenprägung zeigt) von betrügerischer Bankrott, betrügerische Insolven z übernommen: Die österreichische Varietät zeichnet sich also nicht durch eine spezifische Kollokatorenwahl aus. Bei bängiges Verfahren ist zwar die Schweizer Kollokatorenwahl spezifisch, aber sie greift direkt auf einen Helvetismus zurück.

Der dritte Typ von Kollokationen ist schließlich derjenige, der für uns am interessantesten ist: Kollokationen, deren Bausteine in zwei oder mehr Varietäten vorkommen, aber nur in einer davon in kollokatorischer Kombination. Typisch hierfür ist AT regierender Weltmeister, $\mathrm{CH}$ einen Kredit sprechen 
(„einen Kredit bewilligen“), usw. In diesen Kollokationen erfährt (typischerweise) der Kollokator eine varietätenspezifische Bedeutungserweiterung: regierend im Sinne von ,amtierend“, sprechen im Sinne von „bewilligen“ usw.

Die ersten beiden Typen von Kollokationen können mit dem oben in Abschnitt 4.1 beschriebenen Verfahren zur Suche nach Frequenzunterschieden relativ gut identifiziert und „abgeschöpft“ werden: Sie enthalten jeweils mindestens ein Element, das in der betreffenden Varietät signifikant viel häufiger ist als in derjenigen von Deutschland. Wir vergleichen also sowohl die Kollokationen, als auch die in den Kollokationen vorkommenden Einzelwörter nach der Frequenz. Kollokationen, deren eines Element in hohem $\mathrm{Ma}$ varietätentypisch ist, werden separat abgelegt von solchen, wo keiner der Kollokationspartner spezifisch ist. Die Trennung in Realien vs. varietätenspezifische Synonyme zu Wörtern aus dem Gebrauch in Deutschland (Typ AT Krida „Bankrott") muss manuell durchgeführt werden.

\subsection{Korpusbasierte Beschreibung von varietätentypischen Kollokationen}

Fälle von Wörtern, deren Form nur in einer der deutschen Varietäten vorkommt (CH Zustupf „Zuschuss“, AT Krida „Bankrott") sind relativ selten. Die oben genannten Verfahren liefern deswegen in der dritten Teilmenge, d.h. derjenigen der formal „unauffälligen“ Kollokationen immer noch Kandidaten, deren Basen oder Kollokatoren regionale oder nationale Synonyme von Wörtern sind, die in Deutschland üblich sind. Beispiele sind AT bohe Abfertigung (,hohe Abfindung“), ST fixer Professor („fest angestellter Professor") usw. Hier sind Abfertigung und fix in Lesarten benutzt, die regionaltypisch sind. Solche Lesarten bilden in der Regel Reihen innerhalb der jeweiligen Varietät: hohe/ niedrige/ ungerechtfertigte/... Abfertigung, fixer Professor/fixe Stelle/fixe Anstellung/.... Jeweils hat die Basis oder der Kollokator eine relativ stabile varietätentypische Bedeutung. Diese Bedeutung lässt sich für Basen anhand einer geeigneten Kollokationsliste (d.h. der Menge signifikanter Kollokationen mit derselben Basis) manuell ermitteln. Aus dem varietätenspezifischen Korpus können hierzu die häufigsten oder signifikantesten Kollokationskandidaten extrahiert werden; zu CH Anlass („Veranstaltung" finden sich so in unseren Daten (in Reihenfolge abnehmender Varietätenspezifizität (= Quotient der relativen Frequenz)): spezieller Anlass, großer Anlass, kirchlicher Anlass, nationaler Anlass, etc. Die Kollokation sportlicher Anlass ist zwar in unseren Schweizer Texten sehr häufig, aber sie taucht auch in Texten aus Deutschland auf, so dass der „weirdness“-Wert eher gering ist.

Die Ermittlung varietätenspezifischer Kollokatorenbedeutungen setzt einen weiteren Schritt voraus. Ein typisches Beispiel ist eine Schweizer Verwendung des Adjektivs markant. Die von der Frequenz her am stärksten 
vom Gebrauch in Deutschland abweichenden Verwendungen in Schweizer Texten sind in Tabelle 6 angegeben, mit der Absolutfrequenz (größer 7) und dem ,weirdness"-Wert.

\begin{tabular}{|l|c|c|}
\hline \multicolumn{1}{|c|}{ Kollokation } & $\mathbf{f}_{\text {CH }}$ & RF-REL \\
\hline markante Zunahme & 62 & 59,5 \\
markanter Anstieg & 35 & 39,6 \\
markante Steigerung & 31 & 19,8 \\
markante Erhöhung & 9 & 17,3 \\
markante Verbesserung & 40 & inf \\
markante Gewinnsteigerung & 15 & inf \\
\hline markanter Rückgang & 45 & 43,2 \\
markanter Einbruch & 9 & 17,3 \\
markante Abnahme & 10 & inf \\
\hline markante Änderung & 17 & 16,3 \\
\hline markanter Temperatursturz & 8 & inf \\
\hline
\end{tabular}

Tab. 6: Regionale Lesarten von Kollokatoren: markant $_{\mathrm{CH}}$

Die Liste in Tabelle 6 weist Kollokationen auf, in denen markant eine starke (ggf. quantifizierbare) Veränderung eines Werts bezeichnet, etwa einer Lesart des Adjektivs erheblich entsprechend. Dabei sind jeweils die Absolutfrequenz in unseren Schweizer Texten $\left(\mathrm{f}_{\mathrm{ch}}\right)$ und der ,weirdness"-Quotient (RFREL) im Verhältnis zu den Zeitungstexten aus Deutschland angegeben. Ein Vergleich mit den Kollokationen von markant in Texten aus Deutschland, sortiert nach dem Log-Likelihood-Wert (Tabelle 7, zusätzlich mit Angabe der Absolutfrequenz) zeigt, dass die Schweizer Lesart nicht oder nicht prominent vorhanden ist; markant wird mit Nomina kombiniert, die Formen oder Stellen in der Landschaft (Stelle, Gebäude, Bawwerk) bezeichnen.

\begin{tabular}{|l|c|c|}
\hline \multicolumn{1}{|c|}{ Kollokation } & $\mathbf{f}_{\mathrm{DE}}$ & $\mathbf{L L}_{\mathrm{DE}}$ \\
\hline markanter Punkt & 35 & 207,6 \\
markantes Profil & 19 & 145,8 \\
markantes Gesicht & 23 & 133,3 \\
markantes Kinn & 11 & 131,5 \\
markantes Beispiel & 23 & 120,7 \\
markantes Gebäude & 21 & 110,1 \\
markante Stelle & 24 & 103,9 \\
markantes Zeichen & 16 & 93,9 \\
markantes Bauwerk & 9 & 61,9 \\
\hline
\end{tabular}

Tab. 7: Lesarten von Kollokatoren: markant $_{\mathrm{DE}}$ in Texten aus Deutschland 
Ein ähnliches Beispiel stellen die Kollokatoren von Preis in Schweizer und deutschen Texten dar. In Tabelle 8 sind die häufigsten Adjektive mit Preis aufgelistet: in der Reihenfolge abnehmender Häufigkeit in Schweizer Texten, hier angegeben in ,Parts per Million' ( $r f:$ in $\mathrm{ppm}$ ). Neben den Zahlen für das Schweizer Zeitungskorpus sind die analogen Zahlen für ein Teilkorpus des deutschen Zeitungskorpus angegeben, und zwar für 104 Millionen Wörter aus der Zeitung die tageszeitung (Berlin: taz) aus dem Zeitraum 1987 bis 1993.

\begin{tabular}{|l|r|r|}
\hline \multicolumn{1}{|c|}{ Kollokation } & $\mathbf{r f}_{\mathrm{CH}}$ & \multicolumn{1}{c|}{$\mathbf{r f}_{\mathrm{TAZ}}$} \\
\hline hoher Preis & 6,065 & 5,019 \\
günstiger Preis & 4,792 & 0,923 \\
attraktiver Preis & 3,650 & $<0,260$ \\
tiefer Preis & 3,328 & 0,019 \\
erschwinglicher Preis & 0,628 & 0,317 \\
niedriger Preis & 0,546 & 1,740 \\
stolzer Preis & 0,492 & 0,288 \\
\hline
\end{tabular}

Tab. 8: Die Kollokatoren von Preis in Texten aus der Schweiz und aus Deutschland (taz)

Ein Blick auf die Zahlenangaben zeigt erhebliche Unterschiede: Die Schweizer Texte haben proportional häufiger die Kollokationen günstiger Preis, attraktiver Preis und vor allem tiefer Preis. Dagegen ist das aus deutschen Texten vertraute niedriger Preis in den Schweizer Texten seltener als in den deutschen.

Ein Vergleich mit weiteren Kollokationen mit dem Kollokator tief zeigt eine Reihenbildung in den Schweizer Texten: Kombinationen wie tiefe Inflation, tiefer Preis, tiefer Zins/Zinssatz/Zinsniveau sind 40-mal bis 120-mal häufiger in Schweizer Texten als in Texten aus der Bundesrepublik. Zwar gibt es Tief(st)preis(garantie) in der Werbesprache, aber die Kollokation tiefer Preis ist sehr selten: Wir haben nur zwei Belege in unserem Korpus von 258 Millionen Wörtern. Das Adjektiv tief kollokiert in Texten aus Deutschland viel weniger mit Nomina, die den Stand oder Wert von Quantifizierbarem angeben; stattdessen finden sich prominent räumliche Dimensionen (tiefer Graben, tiefes Loch) oder deren übertragene Verwendungsweisen (tiefer Einschnitt, tiefe Spur, tiefe Rezession).

Die detaillierte Interpretation solcher Daten muss interaktiv erfolgen: Ob bei markant und tief spezifische Schweizer Lesarten vorliegen, oder ob die varietätentypischen Kollokationspräferenzen (noch) nicht Anlass geben, eine spezifische Lesart anzusetzen, liegt in der Entscheidung von Lexikografen und Varietätenlinguisten. Aus den Korpusdaten lässt sich jedenfalls für die genannten Fälle mit relativ wenig Aufwand Material extrahieren, welches die Identifikation solcher varietätentypischer Kollokationen auf einfache Weise möglich macht. 
4.4 Das ,tertium comparationis‘: Was bedeuten varietätentypische Kollokationen?

Die oben angegebenen Beispiele für Kollokationen der österreichischen und der Schweizer Nationalvarietät bzw. der Varietät von Südtirol sind intuitiv mit Kollokationen des Deutschen von Deutschland verglichen worden. Wir haben ohne Methodendiskussion, Äquivalente“ in Klammern angegeben und sind davon ausgegangen, dass den Schweizer oder österreichischen Kollokationen auch deutsche Kollokationen, z.B. mit einem anderen Kollokator, entsprechen. Wenn das der Fall ist, kann man sich vorstellen, die Ähnlichkeit (oder: „Äquivalenz") halbautomatisch mit Mitteln der distributionellen Semantik aufzuzeigen (ähnliche Kontexte als Anzeichen für ähnliche Bedeutungen).

Dass in den verschiedenen Varietäten parallel Kollokationen vorliegen, muss aber nicht der Fall sein. Die Kollokationsdaten zum Nomen Information in Texten aus Deutschland liefern folgende Kollokatoren als prominent (in der Reihenfolge absteigender Absolutfrequenz in unserem Korpus): Information sammeln, Information erbalten, Information liefern, Information haben/geben/ austauschen usw. Eine vergleichbare Liste für die Texte der südtiroler Tageszeitung „Dolomiten“ enthält die folgenden Kollokationen, ebenfalls nach Absolutfrequenz absteigend sortiert: Information einholen, Information erteilen, Information geben, Information austauschen, Information erhalten, usw. Bemerkenswert sind Information einholen und Information erteilen. Beide kommen auch in Texten aus Deutschland vor, aber viel seltener: Die Kollokationen sind in den Südtiroler Texten ca. 30-mal häufiger. Zu fragen ist, welcher Ausdruck in Texten aus Deutschland statt des in Südtirol häufigen Information einholen steht.

Eine Detailanalyse der Kontexte von Information einholen zeigt, dass es sich nahezu ausschließlich um Angaben dazu handelt, wo sich der Bürger bei Interesse Informationen verschaffen kann:

Informationen können unter Telefon 0471/63.55.82 eingeholt werden.

Informationen können beim Sekretariat des Skimarathons unter Telefonnummer 0474/97.21.32 eingeholt werden.

Um, äquivalente‘, d.h. texttypologisch und pragmatisch gleichwertige Textstücke aus deutschen Zeitungen zu finden, wurde nach Telefonnummernangaben gesucht. In der „Frankfurter Allgemeinen Zeitung“ finden sich z.B. die folgenden Formulierungen:

Informationen unter Telefon 07541/38.560.

Nähere Informationen gibt es bei der Vertriebsgesellschaft von Aprilia unter der Telefonnummer...

Nähere Informationen sind unter der Telefonnummer ... zu erbalten.

Informationen rund um das Luftbrücken-Jubiläum können unter der Telefonnummer ... abgerufen werden. 
Die Texte aus der FAZ weisen also eine ganz andere grammatische Struktur (z.T. ohne Verb) auf, oder sie verwenden andere Konstruktionen, wie z.B. es gibt. Bei der Untersuchung solcher Unterschiede stößt die automatische Analyse an ihre Grenzen: Hier müssen Parameter berücksichtigt werden, die nicht auf automatischem Wege aus den Texten extrahiert werden können.

\subsection{Varietätenunterschiede bei morphosyntaktischen Präferenzen?}

Oben war darauf hingewiesen worden (vgl. Abschnitt 3.1.2), dass manche Kollokationen deutliche morphosyntaktische Präferenzen aufweisen, d.h. formal weitgehend fixiert sind. Wenn die Kollokatorenwahl varietätenspezifisch sein kann (wie dies im vorigen Abschnitt an Beispielen gezeigt worden ist), dann stellt sich die Frage, ob es zwischen den deutschen Varietäten auch Unterschiede hinsichtlich der morphosyntaktischen Präferenzen einzelner Kollokationen gibt.

Deutlich ist, dass varietätentypische Kollokationen mit regionalspezifischen Basen (z.B. AT das Auslangen finden, ,(s)ein Auskommen finden ${ }^{6}$ oder $\mathrm{CH}$ Einsitz, nebmen, Abgeordneter sein) auch spezifische Präferenzen hinsichtlich der Determination haben (z.B. die Präferenz für den definiten Artikel bei das Auslangen finden). Viel weniger deutlich ist, ob ein und dieselbe Kollokation je nach Varietät mit unterschiedlichen morphosyntaktischen Präferenzen auftritt. Ein solches Beispiel wurde für den Unterschied zwischen portugiesischem und brasilianischem Portugiesisch bei ter ciumes (,neidig sein', wörtlich ,Neid haben', Plural, PT/PT) vs. ter ciume (Singular, PT/BR) in den Textkorpora der Linguateca gefunden.

Aus unseren Daten ergeben sich keine signifikanten Unterschiede hinsichtlich der morphosyntaktischen Präferenzen. Einzelfälle ändern hier wenig am Gesamtbild; die Kollokation Comeback + geben findet sich z.B. in unseren Texten mit wechselndem Determinierer: mit indefinitem Artikel, ein Comeback geben, Possessivum, sein/ibr Comeback geben und in Sonderfällen (in der Regel mit Relativsatz oder einer anderen Ergänzung, die Definitheit erzwingt) mit definitem Artikel, das Comeback geben. Die Texte aus Deutschland und Südtirol weisen eine parallele Verteilung auf: Etwas mehr als die Hälfte der Belege sind indefinit (53-55\%), etwas weniger als die Hälfte haben ein Possessivum (43-47\%). In unseren Schweizer Texten ist demgegenüber der Possessiv-Fall überrepräsentiert: Er macht 63\% der 418 beobachteten Okkurrenzen aus, während nur 33\% indefinit sind. Es mag also sein, dass die Schweizer Autoren eher sein Comeback geben sagen, als ein Comeback geben.

Wir haben aber in unseren Texten ansonsten kaum Fälle gefunden, wo sich solche Präferenzen für einzelne Varietäten aufweisen lassen. Vermutlich gehört die morphosyntaktische Präferenz so eng zum idiomatischen Charakter der Kollokationen, dass sie keinen oder nur sehr geringen Variationen unterliegt. Die Untersuchung weiterer Materialien wäre hier sicher 
angebracht: Erst eine systematische Untersuchung auf der Grundlage sehr großer Textmengen kann hier genügend Daten liefern, so dass eindeutige Aussagen gemacht werden könnten.

\section{Aktueller Stand und weiterer Forschungsbedarf}

In diesem Artikel haben wir versucht, Fragestellungen zu varietätentypischen Kollokationen zu untersuchen. Eine grundlegende Frage war, ob sehr einfache, auf einem Vergleich der relativen Frequenz von Wörtern und Wortkombinationen beruhende Werkzeuge bereits Anhaltspunkte für varietätentypische Kollokationen geben. Die hier referierten Zwischenergebnisse legen die Annahme nahe, dass sich tatsächlich mit solchen einfachen Werkzeugen Material für eine Beschreibung der Kollokationen der deutschen Varietäten extrahieren lässt.

Dabei werden die Kollokationen einer Basis zwischen den zu untersuchenden Varietäten verglichen; ergeben sich Unterschiede bei der Kollokatorenwahl, so lohnt es sich, auch weitere typische Basen eines gegebenen varietätenspezifischen Kollokators zu identifizieren, um eine eventuelle Reihenbildung (z.B. bei CH markant) festzustellen. Die Datenbank, die mit unseren Extraktionsergebnissen befüllt wird, eignet sich für die teilweise Automatisierung solcher Vergleiche relativ gut.

Aus den ersten Experimenten ist deutlich geworden, dass die deutschen Nationalvarietäten zum Teil unterschiedliche Präferenzen hinsichtlich der Kollokatoren haben: Was in Deutschland niedriger Preis heißt, findet sich in Schweizer Texten eher als tiefer Preis. Gleichzeitig legen die hier beschriebenen Experimente die Annahme nahe, dass die morphosyntaktischen Präferenzen bei Kollokationen (d.h. Numerus, Determination usw.) quer durch die Varietäten relativ stabil sind - vielleicht weil sie Teil der Idiomatisiertheit der Kollokationen sind. Allerdings sollten hier mehr Untersuchungen vorgenommen werden, an größeren und texttypologisch sauberer klassifizierten Texten; erst auf der Basis breiterer Untersuchungen kann hier ein abschließendes Urteil gefällt werden.

Der Gegenstandsbereich ist mit den hier vorgelegten Untersuchungen noch keineswegs zufriedenstellend behandelt: Unsere bisherigen Arbeiten konnten noch nicht ausreichend in die Tiefe und in die Breite gehen, sondern stellen eher Stichproben und Experimente zum Test der Werkzeuge dar. Eine systematische Untersuchung wäre sicher sinnvoll. Hierbei ist festzustellen, dass nicht nur die deutschen Nationalvarietäten, sondern auch andere pragmatische Aspekte der Sprache zu untersuchen wären: auch über registerspezifische Kollokationen, über die Texttypgebundenheit bestimmter Kollokationen (z.B. ,Amtssprache) usw. ist wenig bekannt. Die Werkzeuge sind für diese Art Fragestellungen gleichermaßen einsetzbar. 
Längerfristig hoffen wir, dass sich auf der Grundlage großer Textkorpora und effektiver Extraktionsmethoden weitere Details untersuchen lassen; eine Erweiterung der Abdeckung bestehender Variantenwörterbücher im Bereich der Kollokationen wäre sicher für die Dokumentation des Deutschen in seinen vielen verschiedenen Erscheinungsformen nützlich.

\section{Literatur}

Ahmad, Khurshid et al. (1992): What is a term? The semi-automatic extraction of terms from text. In: Snell-Hornby, Mary et al. (Hg.): Translation studies - an interdiscipline. (= Benjamins Translation Library 2). Amsterdam/Philadelphia, S. 267-278.

Ammon, Ulrich (1995): Die deutsche Sprache in Deutschland, Österreich und der Schweiz: das Problem der nationalen Varietät. Berlin/New York.

Ammon, Ulrich et al. (2004): Variantenwörterbuch des Deutschen. Die Standardsprache in Österreich, der Schweiz und Deutschland sowie in Liechtenstein, Luxemburg, Ostbelgien und Südtirol. Berlin u.a.

Bartsch, Sabine (2004): Structural and functional properties of collocations in English. A corpus study of lexical and pragmatic constraints on lexical co-occurence. Tübingen.

Dunning, Ted (1993): Accurate methods for the statistics of surprise and coincidence. In: Computational Linguistics 19, 1, S. 61-74.

Dykstra, Anne/Schoonheim, Tanneke (Hg.) (2010): Proceedings of the XIVth EURALEX International Congress (Leeuwarden, 6-10 July 2010). Leeuwarden. [CD-ROM].

Evert, Stefan (2004): The statistical analysis of morphosyntactic distributions. In: Lino, Maria Teresa et al. (Hg.): Proceedings of the $4^{\text {th }}$ International Conference on Language Resources and Evaluation (LREC), Lisbon, Portugal 2004. Paris, S. 1539 1542.

Evert, Stefan (2005): The statistics of word cooccurrences - word pairs and collocations. Stuttgart. Internet: www.collocations.de (Stand: 15.12.2010).

Fazly, Afsaneh/Stevenson, Suzanne (2006): Automatically constructing a lexicon of verb phrase idiomatic combinations. In: Proceedings of the Workshop on Multiwords in a Multilingual Context, $11^{\text {th }}$ Conference of the European Chapter of the Association for Computational Linguistics, Trento, Italy, April 2006. Trient, S. 337-344.

Forkl, Yves (2010): Zur digitalen Zukunft der Kollokationslexikographie, Perspektiven der Präsentation von Wissen über usuelle französische und deutsche Wortverbindungen in gedruckten und elektronischen Wörterbüchern. Münster.

Hausmann, Franz Josef (1979): Un dictionnaire des collocations est-il possible? In: Travaux de Linguistique et de Littérature XVII, 1, S. 187-195.

Hausmann, Franz Josef (1984): Wortschatzlernen ist Kollokationslernen. In: Praxis des neusprachlichen Unterrichts 31, 4, S. 395-406. 
Hausmann, Franz Josef (2004): Was sind eigentlich Kollokationen? In: Steyer (Hg.), S. 309-334.

Heid, Ulrich (1994): On ways words work together - topics in lexical combinatorics. In: Martin, Willy et al. (Hg.): Proceedings of the VIth Euralex International Congress. Amsterdam, S. 226-257.

Heid, Ulrich (1998): Towards a corpus-based dictionary of German noun-verb collocations. In: Proceedings of the Euralex International Congress 1998. Liège, S. $301-$ 312.

Heid, Ulrich (2009): La saisie des collocations: objectifs et approaches. In: Cahiers de Lexicologie 94, S. 71-89. [Sonderband: Le traitement automatique du lexique, hrsg. v. Blumenthal, Peter/Petit, Gerard].

Heid, Ulrich et al. (2008): Providing corpus data for a dictionary for German juridical phraseology. In: Storrer, Angelika et al. (Hg.): Text resources and lexical knowledge. Selected papers from the $9^{\text {th }}$ Conference of Natural Language Processing, KONVENS 2008. (= Text, Translation, Computational Processing 8). Berlin u.a., S. 131-144.

Heid, Ulrich/Weller, Marion (2010): Corpus-derived data on German multiword expressions for lexicography. In: Dykstra/Schoonheim (Hg.), S. 331-340. [CD-ROM].

Ivanova, Kremena et al. (2008): Evaluating a German sketch grammar: a case study on noun phrase case. In: Proceedings of LREC-2008, Linguistic Resources and Evaluation Conference. Marrakesch. [CD-ROM].

Kilgarriff, Adam et al. (2004): The Sketch Engine. In: Williams, Geoffrey/Vessier, Sandra (Hg.): Proceedings of the 11th Euralex International Congress, Lorient, France, July 6-10, 2004. Bd. 1. Lorient, S. 105-115.

Kilgarriff, Adam et al. (2010): A quantitative evaluation of word sketches. In: Dykstra/ Schoonheim (Hg.), S. 372-379. [CD-ROM].

Krenn, Brigitte (2000): The usual suspects: data-oriented models for the identification and representation of lexical collocations. Diss. Saarbrücken.

Mel'čuk, Igor A. et al. (1984): Dictionnaire explicatif et combinatoire du français contemporain. Bd. I-IV. (= Recherches Lexico-Sémantiques 1). Montréal.

Schiehlen, Michael (2003): A cascaded finite-state parser for German. In: Proceedings of EACL 2003. Bd. 2. Budapest, S. 163-166.

Smadja, Frank (1993): Retrieving collocations from text. In: Computational Linguistics 19, 1, S. 143-177.

Steyer, Kathrin (Hg.) (2004): Wortverbindungen - mehr oder weniger fest. (= Jahrbuch des Instituts für Deutsche Sprache 2003). Berlin/New York.

Tutin, Agnès (2010): Sens et combinatoire lexicale: de la langue au discours. Habil. Grenoble. 
